\title{
From Salt in Solution to Solely Ions - Solvation of Methyl Viologen in Deep Eutectic Solvents and Ionic Liquids
}

Jeffrey M. Klein ${ }^{\dagger}$, Henry Squire†, William Dean, Burcu E. Gurkan*

Department of Chemical Engineering Biomolecular Engineering, Case Western Reserve University, 10900 Euclid Avenue, Cleveland, Ohio, USA 44106

$\dagger$ Equal contribution

*beg23@,case.edu

\section{Supplementary Information}

Table S1: Water contents for samples measured by KF titrator

\begin{tabular}{|l|l|}
\hline Solvent System & Water Content (ppm) \\
\hline$\left[\mathrm{PYR}_{13}\right][\mathrm{TFSI}]$ & 86.4 \\
& $110^{*}$ \\
\hline $10 \mathrm{mM}$ MV in $\left[\mathrm{PYR}_{13}\right][$ TFSI] & 364 \\
& $547^{*}$ \\
\hline $1: 2 \mathrm{ChCl}:$ EG & 76 \\
& $476^{*}$ \\
\hline $1: 4$ ChCl:EG & 123 \\
& --- \\
\hline $1: 6$ ChCl:EG & 105 \\
& --- \\
\hline 10 mM MV in 1:2 ChCl:EG & 325 \\
& $480^{*}$ \\
\hline $1: 2$ ChTFSI:EG & 829 \\
& $3611^{*}$ \\
\hline $1: 4$ ChTFSI:EG & 1078 \\
& $2296^{*}$ \\
\hline $1: 6$ ChTFSI:EG & 1807 \\
& $6235^{*}$ \\
\hline 10 mM MV in 1:2 ChTFSI:EG & 1520 \\
& --- \\
\hline
\end{tabular}

*The water contents were measured after Raman experiments 

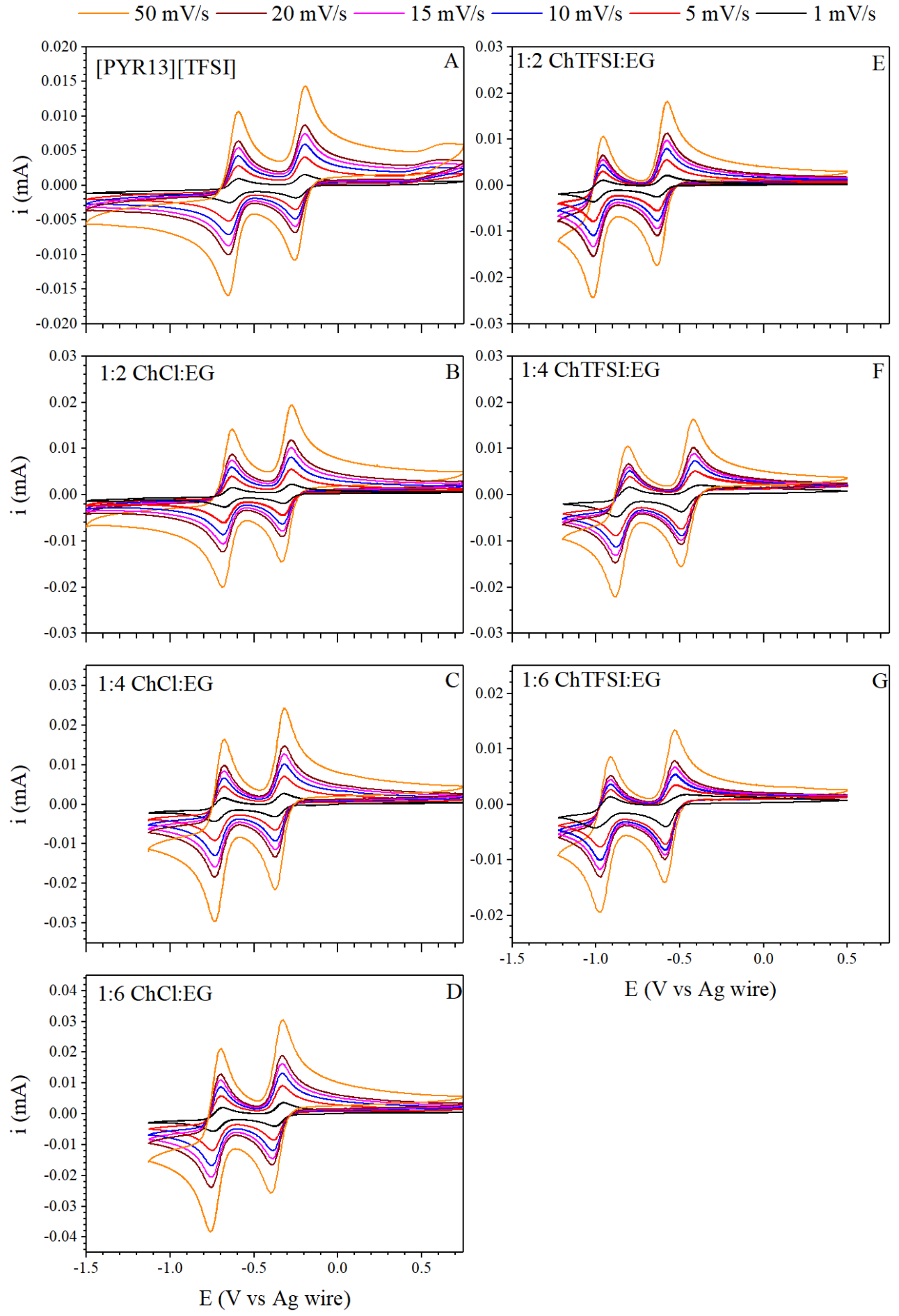

Figure S1: CVs of $10 \mathrm{mM} \mathrm{MVCl}_{2}$ dissolved in (A) [PYR $\mathrm{MP}_{13}$ [TFSI], (B) 1:2 ChCl:EG, (C) 1:4 ChCl:EG, (D) 1:6 ChCl:EG, (E) 1:2 ChTFSI:EG, (F) 1:4 ChTFSI:EG, and (G) 1:6 ChTFSI:EG. CVs were recorded for 5 cycles at the following scan rates: $1,5,10,15,20$, and $50 \mathrm{mV} / \mathrm{s}$, the last cycle is shown for each scan rate. 

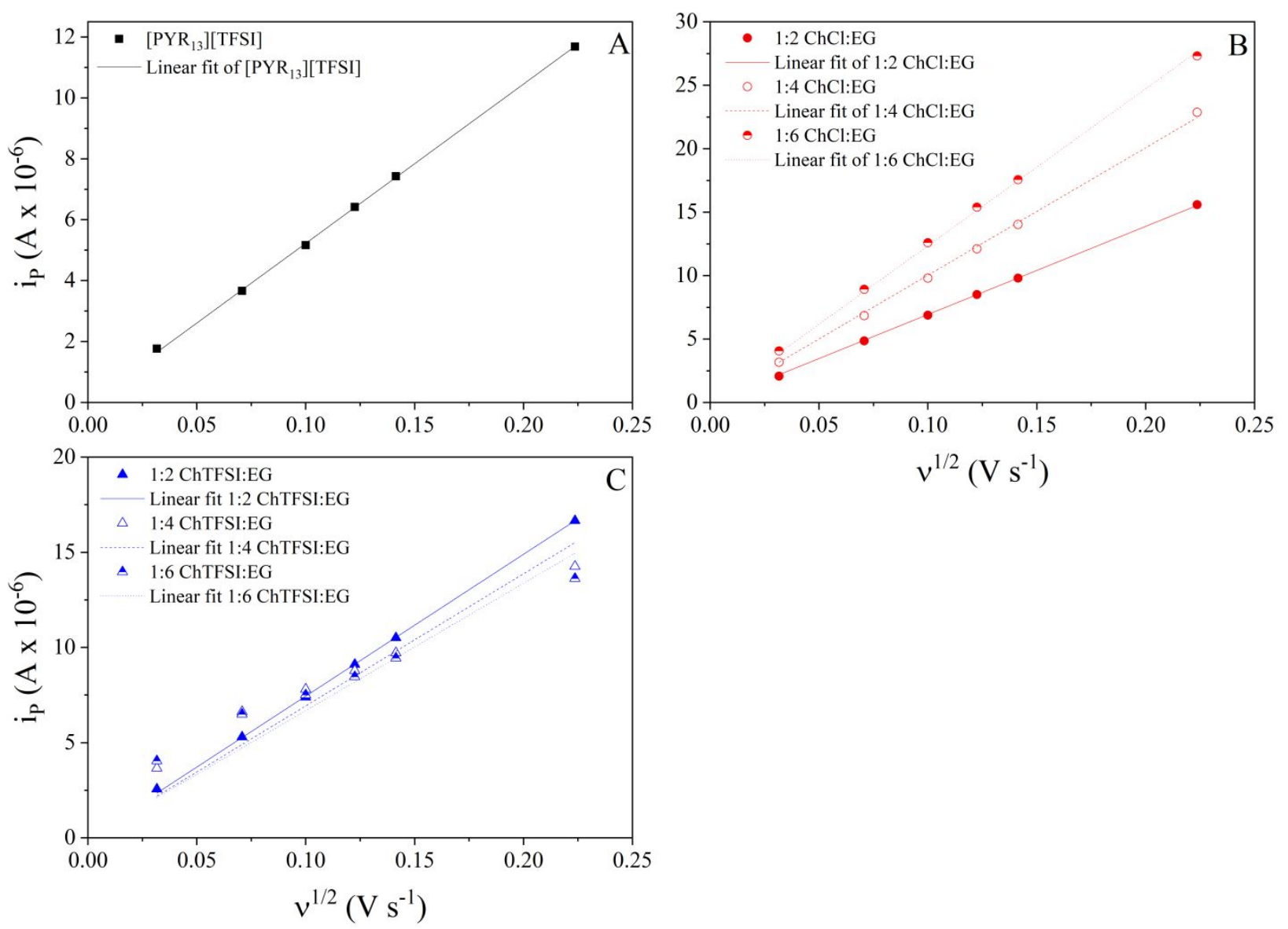

Figure S2: Extracted peak currents from the measured CV's as a function of scan rate ${ }^{1 / 2}\left(v^{1 / 2}\right)$. The linear fits represent the Randles-Sevcik (RS) equation. The IL in (A) and mixtures of ChCl:EG in (B) show exceptional agreement with RS over all scan rates. The ChTFSI:EG mixtures in (C) are not modelled well with RS over all scan rates, possibly due to the buildup of $\mathrm{MV}^{+}$in the system during cycling. Due to the buildup of color near the electrode during cycling, the diffusion coefficients for all systems were determined at $1 \mathrm{mV} / \mathrm{s}$, and not from the slope of RS line. 
Table S2: Measured physical properties for neat solvents as a function of temperature; (A) density, (B) conductivity, (C) viscosity. The density and viscosity data used for ethaline on the Walden analysis are elsewhere (Zhang et al. ${ }^{46}$ )

(A)

\begin{tabular}{|l|l|l|l|l|l|l|}
\hline \multicolumn{7}{|l|}{ Density $\left(\mathbf{g} / \mathbf{c m}^{3}\right)$} \\
\hline $\mathrm{T}\left({ }^{\circ} \mathrm{C}\right)$ & $\begin{array}{l}{[\mathrm{PYR}} \\
{[\mathrm{TF}]}\end{array}$ & $\begin{array}{l}1: 4 \\
\text { ChCl:EG }\end{array}$ & $\begin{array}{l}1: 6 \\
\text { ChCl:EG }\end{array}$ & $\begin{array}{l}1: 2 \\
\text { ChTFSI:EG }\end{array}$ & $\begin{array}{l}1: 4 \\
\text { ChTFSI:EG }\end{array}$ & $\begin{array}{l}1: 6 \\
\text { ChTFSI:EG }\end{array}$ \\
\hline 25 & 1.42784 & 1.11390 & 1.11374 & 1.38490 & 1.31959 & 1.27881 \\
\hline 30 & 1.42331 & 1.11088 & 1.11061 & 1.38057 & 1.31539 & 1.27476 \\
\hline 35 & 1.41881 & 1.10786 & 1.10747 & 1.37621 & 1.31117 & 1.27068 \\
\hline 40 & 1.41432 & 1.10484 & 1.10434 & 1.37183 & 1.30695 & 1.26659 \\
\hline 45 & 1.40985 & 1.10182 & 1.10121 & 1.36743 & 1.30271 & 1.26248 \\
\hline 50 & 1.40540 & 1.09880 & 1.09807 & 1.36302 & 1.29845 & 1.25835 \\
\hline 55 & 1.40097 & 1.09578 & 1.09495 & 1.35858 & 1.29411 & 1.25420 \\
\hline
\end{tabular}

(B)

\begin{tabular}{|l|l|l|l|l|l|l|l|}
\hline \multicolumn{2}{|l|}{ Conductivity (mS/cm) } \\
\hline $\mathrm{T}\left({ }^{\circ} \mathrm{C}\right)$ & $\begin{array}{l}{[\mathrm{PYR}} \\
{[\mathrm{TF}]}\end{array}$ & $\begin{array}{l}1: 2 \\
\text { ChCl:EG }\end{array}$ & $\begin{array}{l}1: 4 \\
\text { ChCl:EG }\end{array}$ & $\begin{array}{l}1: 6 \\
\text { ChCl:EG }\end{array}$ & $\begin{array}{l}1: 2 \\
\text { ChTFSI:EG }\end{array}$ & $\begin{array}{l}1: 4 \\
\text { ChTFSI:EG }\end{array}$ & $\begin{array}{l}1: 6 \\
\text { ChTFSI:EG }\end{array}$ \\
\hline 25 & 3.31 & 7.63 & 9.78 & 7.80 & 3.60 & 4.02 & 3.13 \\
\hline 30 & 3.99 & 9.14 & 11.10 & 9.16 & 4.42 & 4.94 & 3.81 \\
\hline 35 & 4.74 & 10.77 & 12.76 & 10.50 & 5.32 & 6.00 & 4.56 \\
\hline 40 & 5.55 & 12.58 & 14.60 & 12.10 & 6.35 & 7.15 & 5.40 \\
\hline 45 & 6.44 & 14.52 & 16.58 & 13.71 & 7.45 & 8.39 & 6.31 \\
\hline 50 & 7.41 & 16.56 & 18.81 & 15.38 & 8.66 & 9.75 & 7.30 \\
\hline 55 & 8.43 & 18.86 & 21.35 & 17.44 & 9.98 & 11.24 & 8.38 \\
\hline
\end{tabular}

(C)

\begin{tabular}{|l|l|l|l|l|l|l|}
\hline \multicolumn{6}{|l|}{ Viscosity (cP) } \\
\hline $\mathrm{T}\left({ }^{\circ} \mathrm{C}\right)$ & $\begin{array}{l}{[\mathrm{PYR} 13]} \\
{[\mathrm{TFSI}]}\end{array}$ & $\begin{array}{l}1: 4 \\
\text { ChCl:EG }\end{array}$ & $\begin{array}{l}1: 6 \\
\text { ChCl:EG }\end{array}$ & $\begin{array}{l}1: 2 \\
\text { ChTFSI:EG }\end{array}$ & $\begin{array}{l}1: 4 \\
\text { ChTFSI:EG }\end{array}$ & $\begin{array}{l}1: 6 \\
\text { ChTFSI:EG }\end{array}$ \\
\hline 25 & 59.21 & 27.81 & 23.56 & 30.56 & 28.99 & 22.27 \\
\hline 30 & 48.27 & 23.32 & 20.16 & 24.82 & 23.44 & 17.90 \\
\hline 35 & 40.11 & 19.38 & 17.33 & 20.46 & 18.97 & 14.46 \\
\hline 40 & 33.85 & 16.64 & 14.72 & 17.15 & 15.74 & 12.12 \\
\hline 45 & 29.01 & 14.40 & 12.71 & 14.48 & 13.28 & 10.36 \\
\hline 50 & 24.95 & 12.65 & 11.22 & 12.28 & 11.32 & 8.85 \\
\hline 55 & 21.53 & 11.14 & 9.680 & 10.70 & 9.77 & 7.73 \\
\hline
\end{tabular}


Table S3: Peak assignments and location for the vibrational modes of $\mathrm{MV}^{2+}, \mathrm{ChCl}, \mathrm{ChTFSI}$, and EG in different solvent systems.

\begin{tabular}{|c|c|c|c|c|c|c|c|c|}
\hline & [PYR 1 $\left._{13}\right]$ [TFSI] & & $\mathrm{hCl}: \mathrm{E}$ & & & TFSI & & \\
\hline Modes & neat & $1: 2$ & $1: 4$ & $1: 6$ & $1: 2$ & $1: 4$ & $1: 6$ & EG \\
\hline MV & & & & & & & & \\
\hline $840(v \mathrm{C}-\mathrm{C})$ & 838 & 842 & 840 & 839 & 838 & 839 & 838 & 839 \\
\hline $1286(v \mathrm{C}-\mathrm{C})$ & - & 1283 & 1287 & 1288 & 1290 & 1291 & 1290 & 1291 \\
\hline 1302 (v Ring) & 1297 & 1298 & 1301 & 1302 & 1303 & 1305 & 1305 & 1306 \\
\hline 1554 (v Ring) & 1537 & 1534 & 1540 & 1540 & 1561 & 1561 & 1561 & 1560 \\
\hline 1657 (v Ring) & 1645 & 1646 & 1647 & 1649 & 1649 & 1649 & 1650 & 1647 \\
\hline ChCl & & & & & & & & \\
\hline 717 (vs C-N) & & 715 & 714 & 714 & & & & \\
\hline 877 (vs C-N) & & 872 & 886 & 871 & & & & \\
\hline $956($ va C-N) & & 955 & 954 & 952 & & & & \\
\hline $1452\left(\omega \mathrm{CH}_{2}\right)$ & & 1453 & 1448 & 1448 & & & & \\
\hline ChTFSI & & & & & & & & \\
\hline 711 (vs C-N) & & & & & 712 & 713 & 713 & \\
\hline $739\left(\delta \mathrm{CF}_{3}\right.$, vs SNS $)$ & & & & & 740 & 741 & 741 & \\
\hline 875 (vs C-N) & & & & & 882 & 883 & 883 & \\
\hline $952($ va C-N) & & & & & 955 & 954 & 956 & \\
\hline $\left.1133(\text { vs SO})_{2}\right)$ & & & & & 1132 & 1131 & 1129 & \\
\hline $1241\left(\right.$ vs $\left.\mathrm{CF}_{3}\right)$ & & & & & 1238 & 1237 & 1238 & \\
\hline $1332\left(\mathrm{va}, \mathrm{op} \mathrm{SO} \mathrm{SO}_{2}\right)$ & & & & & 1342 & 1337 & 1337 & \\
\hline $1450\left(\omega \mathrm{CH}_{2}\right)$ & & & & & 1450 & 1452 & 1451 & \\
\hline EG & & & & & & & & \\
\hline $865(v \mathrm{C}-\mathrm{C})$ & & 863 & 866 & 858 & 867 & 865 & 867 & \\
\hline $1042\left(v \mathrm{CH}_{2}-\mathrm{CH}_{2}-\mathrm{O}\right)$ & & 1054 & 1052 & 1044 & 1051 & 1053 & 1050 & \\
\hline $1069(\delta \mathrm{C}-\mathrm{OH})$ & & 1068 & 1065 & 1064 & 1062 & 1064 & 1060 & \\
\hline $1092\left(\delta, \tau \mathrm{CH}_{2}\right)$ & & 1088 & 1084 & 1090 & 1079 & 1076 & 1090 & \\
\hline & & & & & & 1086 & & \\
\hline $1463\left(\right.$ vs $\left.\mathrm{CH}_{2}\right)$ & & 1464 & 1466 & 1461 & & & & \\
\hline
\end{tabular}




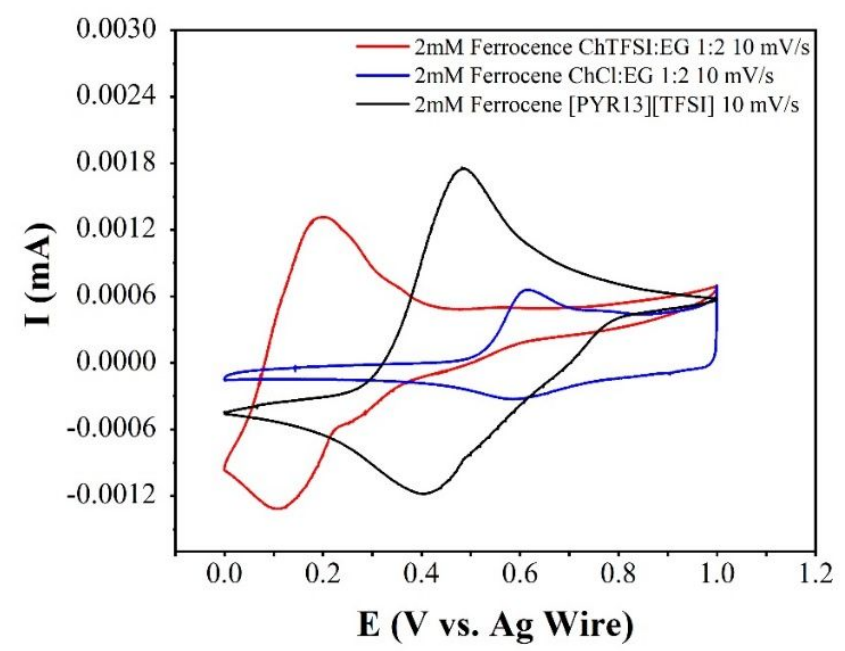

Figure S3: CVs of $2 \mathrm{mM}$ Ferrocene in [PYR 13$]$ [TFSI], ChCl:EG (1:2), ChTFSI:EG (1:2) at 10 $\mathrm{mV} / \mathrm{s}$. CVs correspond to the 10 th cycles. 